\title{
Growth Differentiation Factor 15 in Patients with Acute Coronary Syndrome and Its Relation to Type 2 Diabetes Mellitus \\ Mahmoud Abdelsabour Abdellah ${ }^{1}$, Hosney Ali Hassen², Abdel-Rahim Mohammed Abdel-Hafiz ${ }^{2}$, Doua Ahmed Fouad, Aliaa Aly Youssef*2, Naglaa Kamal Idriss ${ }^{2}$ \\ Departments of ${ }^{1}$ Cardiology and ${ }^{2}$ Medical Biochemistry, Faculty of Medicine, Assuit University \\ *Corresponding author: Aliaa Aly Youssef, Mobile: (+20)01060961596, E-Mail: aliaa.aly1610@gmail.com
}

\begin{abstract}
Background: Growth differentiation factor 15 (GDF15) gene expression level is a novel biomarker that reflects inflammation and oxidative stress in patients with acute coronary syndrome and those complicated with type 2 diabetes mellitus (T2DM). Growth differentiation factor 15 high levels could be a reliable sensitive biomarker for the prediction of acute coronary syndrome.

Patients and Methods: This Cross-sectional/case-control study was conducted at the Cardiology Department, Assiut University during the period from January 2018 to December 2019, and patients were selected with the exclusion of any inflammatory or autoimmune disease.

Results: Growth differentiation factor 15 gene expression was significantly higher in acute coronary syndrome patients compared to the controls ( $\mathrm{p}$-value $<0.001$ ) and its expression was significantly higher in acute coronary syndrome patients with type 2 diabetes mellitus group compared to acute coronary syndrome patient group (pvalue $<0.05$ ). Growth differentiation factor 15 had $92 \%$ sensitivity and $77 \%$ specificity in the prediction of acute coronary syndrome which was statistically significant ( $\mathrm{p}$-value $<0.001$ ), while yielded $73 \%$ sensitivity and $53 \%$ specificity of no statistical significance ( $\mathrm{p}$-value $=0.829$ ) in the prediction of type 2 diabetes mellitus among patients with acute coronary syndrome.

Conclusion: Growth differentiation factor 15 gene expression could be used as a reliable sensitive biomarker for prediction of the acute coronary syndrome but more studies will be mandatory soon to clarify its role as a predictive marker for type 2 diabetes mellitus development among patients with the acute coronary syndrome.

Keywords: Acute coronary syndrome, Type 2 Diabetes Mellitus, Gene Expression, Growth Differentiation Factor 15 prediction.
\end{abstract}

\section{INTRODUCTION}

Acute coronary syndrome (ACS) is the leading cause of death and disability worldwide especially in developing countries, the mortality rate in individuals over 35 years old from CAD compromise one-third of deaths worldwide ${ }^{(1)}$. Growth differentiation factor 15 (GDF15) is a stressresponsive cytokine, a member of the transforming growth factor-beta (TGF $\beta$ ) superfamily. GDF15 is expressed at low levels in all organs, whereas it is expressed in high concentrations in the liver, kidney, heart, and lungs in response to ischemia, oxidative stress, and mechanical stress ${ }^{(2)}$.

GDF15 has been examined as a novel emerging biomarker for $\mathrm{CAD}^{(3)}$. Increased expression of GDF15 was identified in atherosclerotic lesions and within the myocardium in acute myocardial infarction (AMI) ${ }^{(4)}$. GDF15 has been considered as a marker of poor prognosis in patients with CAD ${ }^{(5)}$, acute myocardial infarction, atrial fibrillation, and heart failure ${ }^{(6)}$.

Type 2 diabetes mellitus (T2DM) is an important risk factor for $\mathrm{CAD}$ and hyperglycemia results in oxidative stress with reactive oxygen species (ROS) generation that affect the integrity of the endothelial cells and predispose to cardiovascular complications. GDF15 is very important in the regulation of glucose homeostasis and lipid metabolism, also it improves insulin sensitivity and regulates food intake ${ }^{(7,8)}$.

This study aimed to clarify the pattern of GDF15 gene expression in acute coronary syndrome patients with or without type 2 diabetes mellitus and its role as a predictive biomarker for type 2 diabetes mellitus among those patients.

\section{PATIENTS AND METHODS}

This Cross-sectional /Case-control study was conducted during the period from January 2018 to December 2019. This study included three studied groups; 23 patients with acute coronary syndrome (ACS group), 22 ACS patients with type 2 diabetes mellitus group (ACS with T2DM), and 45 agematched healthy control group. The sample size was calculated using EPi Info 2000, version 1.1.2 (Center for Disease Control and Prevention, Atlanta, Georgia, USA).

This study had been performed at the coronary care unit of the Cardiology Department, Assiut University Hospital. Patients presented by clinical manifestations and ECG changes of acute coronary syndrome were included in the study. All patients were subjected to 
full history taking, meticulous general examination, and local cardiac examination.

\section{Laboratory tests:}

Laboratory investigations including creatine kinase-MB (CK-MB), troponin-T, and lipid profile were performed for all patients at admission as routine laboratory work in the Clinical Pathology Department, Assiut University by the following kits: human CK-MB ELISA kit, Cell Biolabs, USA (Catalog No. PRB-5047-5); human troponin T (Tn-T) ELISA kit, My BioSource, USA (Catalog No. MBS265440) and colorimetric assay kits for lipid profile.

\section{Genetic analysis:}

GDF15 gene expression had been evaluated by quantitative real-time PCR (qPCR), the total RNA was extracted from whole blood collected in EDTA tubes using Direct-zol ${ }^{\text {TM }}$ RNA Miniprep Plus (TRI Reagent ${ }^{\circledR}$ not included) (Catalog No. R2070T) and the extracted RNA stored at $-80^{\circ} \mathrm{C}$ in EL-Raghi Research center, Assiut University Hospital. Complementary DNA (cDNA) synthesis was performed by SensiFAST ${ }^{\mathrm{TM}}$ SYBR ${ }^{\circledR}$ Lo-ROX Kit, USA, (Catalog No. 94005).

The thermal cycler (Applied Biosystems Step One Plus TMReal-Time PCR Systems, California, USA) was programmed to $95^{\circ} \mathrm{C}$ for 10 minutes, followed by 40 cycles of $95^{\circ} \mathrm{C}$ for 5 seconds then 60 $\mathrm{C}$ for 1 minute. Gene expression was normalized to the GABDH housekeeping gene as a reference gene and is reported as fold change gene expression. We used $1 \mu$ of each forward and reverse primers for each gene. The following primers were obtained from (Macrogen,UK) ${ }^{(9)}$.

GDF15, F : 5 'TCAAGGTCGTGGGACGTGACA 3' and

$\mathbf{R}: 5^{\prime}$ GCCGTGCGGACGAAGATTCT 3' ;

GABDH , F : 5' AAGTTCAACGGCAGTCAAG 3' and $\mathbf{R}:$ 5' ACTCAGCACCAGCTCACC 3'

\section{Ethical approval:}

The present study was ethically approved by the Ethical Committee of the Faculty of Medicine, Assiut University.

The purpose and method of the study were briefed to each participant and informed consent was obtained. Patients with autoimmune diseases and cancer were excluded from the study.

\section{Statistical analysis}

The statistical analysis was performed using SPSS version 21.0 (IBM-SPSS, Chicago, IL, USA). Quantitative data were presented as mean \pm SD. Qualitative data were expressed as frequency and percentage. The following tests were done. Independent t-test analysis was carried out to compare the means of normally distributed dichotomous data. Differences between the groups were analyzed by one way ANOVA, followed by post-hoc analysis with Bonferroni's test correction for normally distributed values.

Gene expression profile modulations were evaluated comparing CT values using the $2^{\wedge-\Delta \Delta C t}$ method. Receiving operation characteristic (ROC) curve (analyzed as the area under the curve (AUC), standard error (SE) and 95\% confidence interval (CI) was constructed to detect the statistical validity (sensitivity, specificity). Probability (P-value) $\leq 0.05$ was considered significant.

\section{RESULTS}

There was no statistically significant difference between controls, ACS and ACS withT2DM groups regarding demographic data. Significant differences in the lipid profile were observed between the three studied groups while cardiac enzymes were evaluated in the two patient groups and had no statistically significant differences between both groups (Tables 1, 2 and 3).

Table (1): Demographic and disease history differences between groups

\begin{tabular}{|l|c|c|c|c|}
\hline Parameter & $\begin{array}{c}\text { Controls } \\
(\mathbf{n = 4 5})\end{array}$ & $\begin{array}{c}\text { ACS } \\
(\mathbf{n = 2 3})\end{array}$ & $\begin{array}{c}\text { ACS with T2DM } \\
(\mathbf{n = 2 2})\end{array}$ & \multirow{2}{*}{ P-value } \\
\hline Age (years) & $40.26 \pm 11.1$ & $40.09 \pm 11.8$ & $44.55 \pm 6.9$ & \multirow{2}{*}{0.241} \\
\hline P-value & 1 vs. $2=0.948$ & 2 vs. $3=0.155$ & 1 vs. $3=0.117$ & \\
\hline Sex & & & & - \\
\hline$\bullet$ Female & $32.6 \%$ & $8.7 \%$ & $27.3 \%$ & \\
\hline$\bullet$ Male & $67.4 \%$ & $91.3 \%$ & $72.7 \%$ & $=0.928$ \\
\hline Hypertension & - & $6(26.1 \%)$ & $6(27.3 \%)$ & \multirow{2}{*}{} \\
\hline
\end{tabular}


Table (2): Lipid profile differences between groups

\begin{tabular}{|c|c|c|c|c|}
\hline Parameter & $\begin{array}{c}\text { Controls } \\
(n=45)\end{array}$ & $\begin{array}{c}\text { ACS } \\
(n=23)\end{array}$ & $\begin{array}{l}\text { ACS with T2DM } \\
\qquad(\mathrm{n}=22)\end{array}$ & P-value \\
\hline Cholesterol (mg/dl) & $134.93 \pm 12.1$ & $190.17 \pm 46.3$ & $216.09 \pm 78.7$ & \multirow{2}{*}{$<0.001$} \\
\hline P-value & 1 vs. $2=0.011$ & 2 vs. $3=0.091$ & 1 vs. $3=0.001$ & \\
\hline $\begin{array}{l}\text { TAG } \\
(\mathrm{mg} / \mathrm{dl})\end{array}$ & $92.17 \pm 17.2$ & $110.26 \pm 35.5$ & $98.37 \pm 20.1$ & \multirow[t]{2}{*}{$=0.023$} \\
\hline P-value & 1 vs. $2=0.006$ & 2 vs. $3=0.129$ & 1 vs. $3=0.319$ & \\
\hline $\begin{array}{l}\text { HDL-C } \\
(\mathrm{mg} / \mathrm{dl})\end{array}$ & $36.96 \pm 6.6$ & $39.83 \pm 8.5$ & $43.45 \pm 11.4$ & \multirow[t]{2}{*}{$=0.013$} \\
\hline P-value & 1 vs. $2=0.182$ & 2 vs. $3=0.149$ & 1 vs. $3=0.004$ & \\
\hline $\begin{array}{l}\text { LDL-C } \\
(\mathrm{mg} / \mathrm{dl})\end{array}$ & $108.13 \pm 28.3$ & $129.61 \pm 30.2$ & $131.00 \pm 30.9$ & \multirow[t]{2}{*}{$=0.010$} \\
\hline P-value & 1 vs. $2=0.016$ & 2 vs. $3=0.891$ & 1 vs. $3=0.011$ & \\
\hline
\end{tabular}

TAG: triacylglycerol; HDL-C: high-density lipoprotein cholesterol; LDL-C: low-density lipoprotein cholesterol.

Table (3): Cardiac enzymes differences between patient groups

\begin{tabular}{|l|c|c|c|}
\hline Parameter & $\begin{array}{c}\text { ACS } \\
(\mathbf{n = 2 3})\end{array}$ & $\begin{array}{c}\text { ACS with T2DM } \\
(\mathbf{n = 2 2})\end{array}$ & P-value* \\
\hline CK $(\mathbf{I U} / \mathbf{L})$ & $119.26 \pm 9.1$ & $155.05 \pm 10.2$ & $<\mathbf{0 . 0 0 1}$ \\
\hline CK-MB $(\mathbf{n g} / \mathbf{m l})$ & $78.48 \pm 10.6$ & $73.23 \pm 8.7$ & $=0.702$ \\
\hline Troponin T $(\boldsymbol{\mu g} / \mathbf{L})$ & $0.69 \pm 0.1$ & $1.09 \pm 0.3$ & $=0.314$ \\
\hline
\end{tabular}

CK: creatine kinase; CK-MB: creatine kinase isoenzyme present in myocardium and brain;troponin-T: isoenzyme present in the myocardium.

There was a significant increase in GDF15 fold change gene expression in ACS patient groups compared to the control group while the gene expression was significantly higher in the ACS patients with T2DM group compared to the ACS patient group (Figure 1).

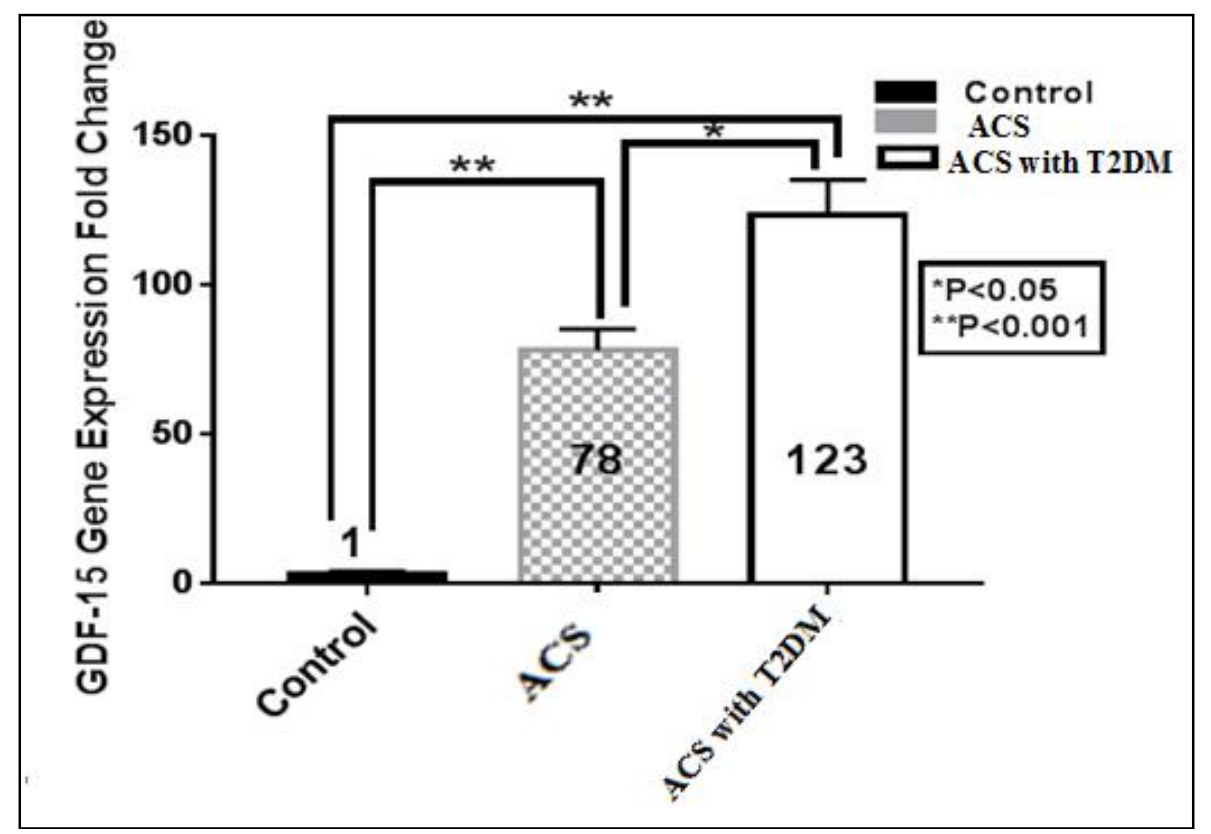

Figure (1): Fold change gene expression $\left(2^{\wedge}-\Delta \Delta \mathrm{Ct}\right)$ forGDF15 in the three studied groups *ANOVA test was used to compare the mean difference between groups, ${ }^{*}$ Post- hoc test with Bonferroni corrections.

ROC curve for GDF15 CT values as a predictive marker for ACS was statistically significant (p- value $<0.001$ ), AUC was (0.926), sensitivity (92\%) and specificity (77\%) (Figure 2). 


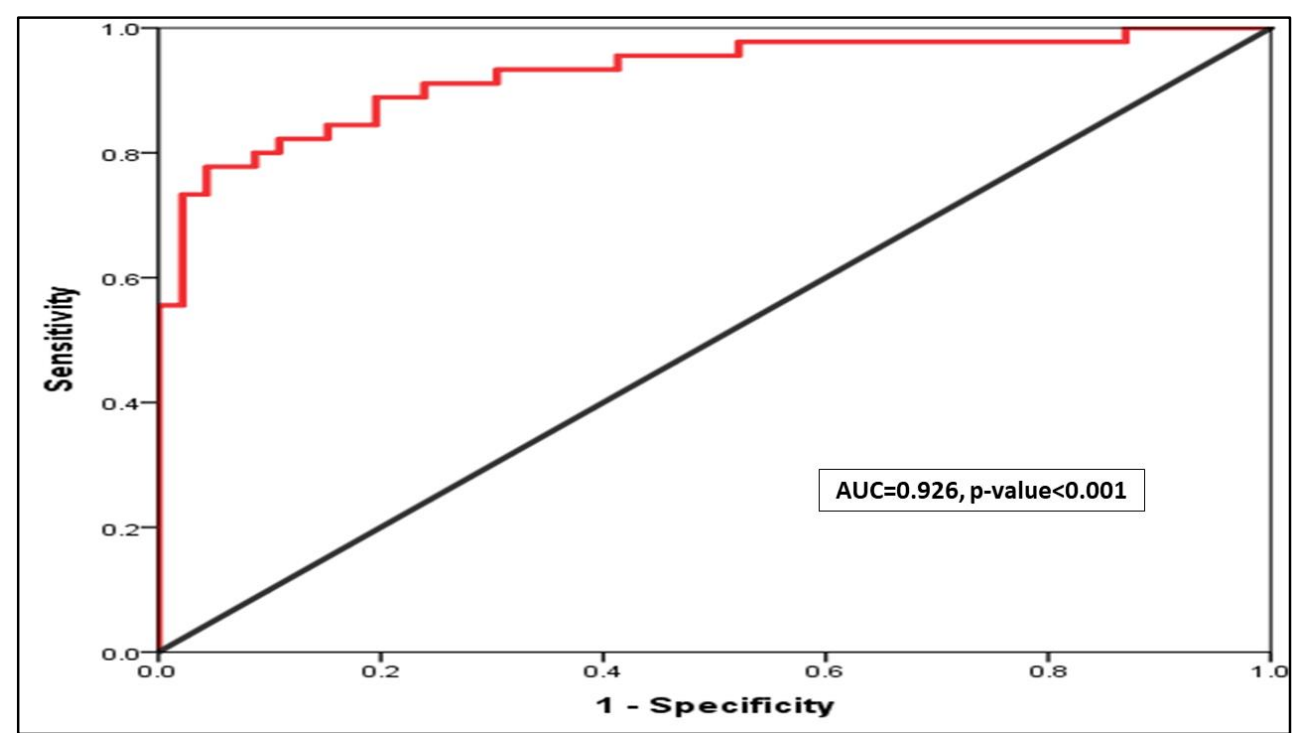

Figure (2): ROC curve for GDF15 gene CT values for prediction of ACS among studied groups (cases versus controls).

ROC curve of GDF15 CT values in prediction of T2DM among ACS patients was statistically non significant (pvalue $=0.829)$, the AUC (0.519), sensitivity (73\%) and specificity (53\%) (Figure 3).

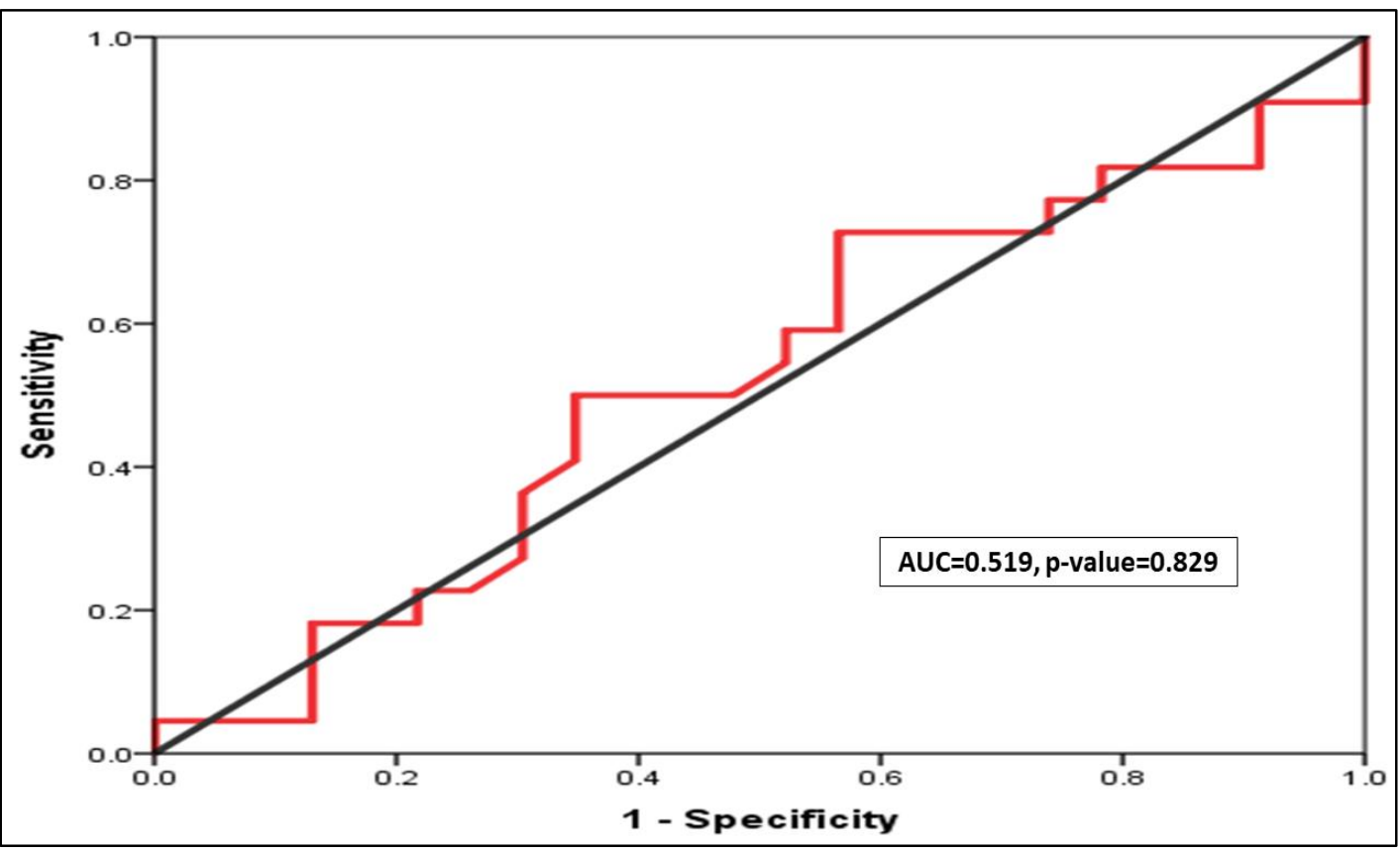

Figure (3): ROC curve of GDF15 gene CT values for prediction of T2DM among all ACS patients. 


\section{DISCUSSION}

Coronary artery atherosclerosis is the most determinant pathology in patients with coronary artery disease, the reduced blood supply progress to a level that causes myocardial infarction and predisposes to heart failure. The most common clinical presentation of coronary artery disease is an acute coronary syndrome (ACS) where the rupture of coronary atherosclerotic plaque with subsequent thrombotic complications are the most serious outcome of it ${ }^{(\mathbf{1 0})}$.

The present study provides a focused synopsis of supposed pathophysiological mechanisms concerned with ACS including GDF15 gene expression level in ACS patients and those complicated with T2DM that has been considered as an important risk factor in those patients.

The current study demonstrated that GDF15 fold change gene expression was significantly high in both ACS patient groups compared to the control group. ROC curve was done for GDF15 gene expression as a predictive marker for ACS development and the results showed that GDF15 is a sensitive and a reliable marker for ACS prediction, it yielded $92 \%$ sensitivity and $77 \%$ specificity. The present results were consistent with Hagström $\boldsymbol{e t}$ al. ${ }^{(5)}$ and Brenière et al. ${ }^{(6)}$ who evaluated the role of GDF15 as a marker of poor prognosis in CAD patients and its increasing level could reflect the presence of cardiac complications such as, atrial fibrillation and heart failure. Although the current study and previous studies considered that high levels of GDF15 is a marker of bad prognosis in CAD patients, other studies showed that GDF15 increased production protects against fatal cardiac rupture in a mouse model with myocardial infarction as it acts as an antiinflammatory cytokine that inhibits both leukocyte integrin and represses myeloid cell recruitment in cardiac muscle after myocardial infarction ${ }^{(11)}$.

GDF15 had been studied as adipokine due to its dramatic impact on glucose and lipid metabolism ${ }^{(7,8,12)}$. Some studies evaluate GDF15 levels in patients with impaired fasting blood glucose concentration and concluded that it is a reliable biomarker for diagnosis and can predict future development of T2DM ${ }^{(13,14)}$. Although the present study showed a significant increase in the fold change gene expression in the ACS patient group with T2DM compared to both controls and ACS patient group, the ROC curve in this study showed that GDF15 gene expression couldn't be used as a reliable sensitive biomarker in the prediction of T2DM among patients with ACS and more studies are needed for further clarification.

The protective and anti-apoptotic role of increased GDF15 levels on endothelial cells in patients with T2DM had been evaluated, hyperglycemia of diabetes results in oxidative stress, ROS generation, and overproduction of proinflammatory cytokines within the vessel wall that lead to apoptosis of endothelial cells ${ }^{(15)}$. GDF 15 overexpression in the macrophages infiltrating these endothelial cells in response to proinflammatory cytokines will protect endothelial cells from apoptosis through increased transcription of antiapoptotic genes $\mathrm{Bcl}-\mathrm{XL}$ and p53 important for the protection of endothelial cells in the atherosclerotic blood vessels and during hyperglycemic conditions that affect the endothelial integrity ${ }^{(16,17)}$.

Although GDF15 appears to be protective for endothelial cells during the hyperglycemic state of diabetes mellitus, its overexpression is still linked to increased oxidative stress, inflammation, and endothelial dysfunction in diabetic patients ${ }^{(18,19)}$. The present study has been considered as the first study that evaluate GDF15 gene expression in periphral blood samples of ACS patients and eludicate T2DM and its impact on GDF15 gene expression profile.

\section{CONCLUSION}

Growth differentiation factor 15 gene expression could be used as a reliable sensitive biomarker for prediction of the acute coronary syndrome but more studies will be mandatory soon to clarify its role as a predictive marker for type 2 diabetes mellitus development among patients with the acute coronary syndrome.

\section{REFERENCES}

1. Benjamin E, Blaha M, Chiuve $S$ et al. (2017): Heart disease and stroke statistics-2017 update. Am Heart Assoc., 8(3):72-78.

2. Xu X, Li Z, Gao W (2011): Growth differentiation factor 15 in cardiovascular diseases: from bench to bedside. Biomarkers, 16(6):466-475.

3. Dalos D, Spinka G, Schneider M et al. (2019): New cardiovascular biomarkers in ischemic heart diseaseGDF-15, A Probable Predictor for Ejection Fraction. Journal of Clinical Medicine, 8(7):924.

4. Kempf T, Eden M, Strelau J et al. (2006): The transforming growth factor- $\beta$ superfamily member growth-differentiation factor- 15 protects the heart from ischemia/reperfusion injury. Circulation Research, 98(3):351-360.

5. Hagström E, Held C, Stewart R et al. (2017): Growth differentiation factor 15 predicts all-cause morbidity and mortality in stable coronary heart disease. Clinical Chemistry, 63(1):325-333.

6. Brenière C, Méloux A, Pédard $M$ et al. (2019): Growth differentiation factor-15 (GDF-15) is associated with mortality in ischemic stroke patients treated with acute revascularization therapy. Frontiers in neurology, 10(1):611-617.

7. Macia L, Tsai V, Nguyen A et al. (2012): Macrophage inhibitory cytokine 1 (MIC-1/GDF15) decreases food intake, body weight and improves glucose tolerance in mice on normal \& obesogenic diets. PloS One, 7(4):34868-72. 
8. Kaur J (2014): A comprehensive review on metabolic syndrome. Cardiology Research and Practice, 14(4): 2090-8016.

9. Yong A, Zeng Y, Vindivich D et al. (2015): Morphological effects on expression of growth differentiation factor 15 (GDF15), a Marker of Metastasis. J Cell Physiol., 229(3): 362-373.

10. Bahnasawy M, Habbak L, Al-Maie M et al. (2013) : Risk factors for coronary artery disease in Egyptian women. The Egyptian Journal of Hospital Medicine, 53(1):827-836.

11. Kempf T, Zarbock A, Widera C et al. (2011): GDF15 is an inhibitor of leukocyte integrin activation required for survival after myocardial infarction in mice. Nature Medicine, 17(5):581-588.

12. Shimano M, Ouchi N, Walsh K (2012): Cardiokines: recent progress in elucidating the cardiac secretome. Circulation, 126(21):327-332.

13. Hong J, Chung H, Park H et al. (2014): GDF15 is a novel biomarker for impaired fasting glucose. Diabetes \& Metabolism Journal, 38(6):472-479.

14. Kempf T, Guba-Quint A, Torgerson J et al. (2012): Growth differentiation factor 15 predicts future insulin resistance and impaired glucose control in obese nondiabetic individuals: results from the XENDOS trial. European Journal of Endocrinology, 167(5):671678.

15. Hingorani A, Cross J, Kharbanda $R$ et al. (2000): Acute systemic inflammation impairs endotheliumdependent dilatation in humans. Circulation, 102(9):994-999.

16. Chrysovergis $K$, Wang $X$, Kosak J et al. (2014): NAG-1/GDF-15 prevents obesity by increasing thermogenesis, lipolysis and oxidative metabolism. International Journal of Obesity, 38(12):1555-8.

17. Zhang Y, Moszczynski LA, Liu Q et al. (2017): Over-expression of growth differentiation factor 15 (GDF15) preventing cold ischemia-reperfusion (I/R) injury in heart transplantation through Foxo3a signaling. Oncotarget, 8(22):36531-4.

18. Kempf T, Von Haehling S, Peter T et al. (2007): Prognostic utility of growth differentiation factor-15 in patients with chronic heart failure. Journal of the American College of Cardiology, 50(11):1054-1060.

19. Ho J, Hwang S, Wollert K et al. (2013): Biomarkers of cardiovascular stress and incident chronic kidney disease. Clinical Chemistry, 59(11):1613-1620. 